\title{
Incentives to Cooperate in Network Formation
}

\author{
HAYDÉE LUGO* and RAÚL JIMÉNEZ \\ Dept. Cómputo Científico y Estadística, Univ. Simón Bolívar, A.P 89000 Caracas - Venezuela; \\ E-mail:hlugo@usb.ve
}

Accepted 17 January 2006

\begin{abstract}
We propose a simple mechanism based on taxes and subsidies to enhance high cooperation in evolutionary networks. The interactions among agents are based on the Spatial Prisoners' Dilemma game in which each agent interacts with the same strategy with its local neighbors, collects an aggregate payoff and imitates the strategy of its best neighbor. First we study the mechanism in a regular lattice where it is well-known that the asymptotic fraction of cooperators fluctuates around a constant value for almost all starting proportions and configurations of cooperators. We also explore the mechanism in random and adaptive networks, where adaptability refers to the ability of agents to change their local neighborhood. For theses networks it has been reported that the starting proportion of cooperation has to be sufficiently high in order to obtain highly cooperative levels in the longrun time. The implementation of our mechanism produces successful results in such evolutionary networks, higher levels of cooperation are reached for all initial fractions of cooperation, including the most adverse case of just one cooperator in the network. Additionally, we observe that the network reaches a spatial configuration such that the fraction of cooperators remains in high level even when the mechanism is switched off. As a result the mechanism can work as an ignition engine to achieve highly cooperative level since it can be implemented for a finite period of time.
\end{abstract}

Key words: Spatial Prisoner's Dilemma, tax-subsidy mechanism, emergence of cooperation

\section{Introduction}

The one-shot two-person Prisoners' Dilemma game is perhaps the most popular model that has been used as the basis for many studies that try to give explanations of how and why cooperation can emerge or persist in social, biological and economic systems. The reason for selecting the PD game is due to its simplicity in showing how a dominant individualistic action leads to the most inefficient collective outcome when all individuals adopt it. In the literature one can find extensions of this game that lead to different results in which cooperation can be observed as a collective phenomena. For instance, the Iterated PD, the Spatial PD, the Continuous PD and combinations of them can be viewed as representing mechanisms that promote cooperation. Doebeli and Hauert (2005) make a review of such models of cooperation based on the PD game. In the Iterated PD game individuals meet repeatedly. As a result cooperation can be obtained when one considers strategical 
complexities or memories of past encounters. Thus, the successful strategies are those that embody the emergence or maintenance of cooperation. The most famous example of this type of strategies is the "Tit-for-Tat" in which individuals cooperate on the first move and then cooperate or defect exactly as the other player did on the preceding move. The direct reciprocity based on retaliation, reputation and trust plays a fundamental goal in promoting cooperative behavior.

When the Iterated PD game does not require memory the effect of spatial structures may be considered. In this context, Nowak and May (1992) introduce a spatial version of the Prisoner's Dilemma game (SPD) in which agents are placed on a regular lattice, interact with their neighbors, collect an aggregate payoff and imitate the strategy of their most successful neighbor. The SPD in a lattice shows chaotically changing spatial patterns in which individuals who always cooperate coexist indefinitely with individuals that always defect. This rich spatiotemporal dynamics reaches an asymptotic overall fraction of cooperators that fluctuates around a constant value Nowak and May (1993). The SPD and its extensions, including agents on small world and random network, has been widely explored in computational simulations to study the complexity of cooperation (see Abramson and Kuperman (2001); Lindgren and Nordahl (1994) and Zimmermann et al. (2001)).

Very recently, a subtle point of view in modeling social system refers to the issue that individuals are able not only to modify their actions but also to modify their interactions. Since the endogeneity is an ubiquitous feature of real social interactions the question to be answered is whether cooperation could be sustained or could enhance higher levels when endogenously determined interactions exist. Vriend (2005) presents an overview of Agent-based Computational Economics models that cover a wide range of possibilities to model endogenous interactions in many different contexts. However in the context of social dilemmas there is a limited number of works that has explored such that kind of dynamics. For instance, Hanaki et al. (2005) and Eguíluz et al. (2005) tackle the problem of how cooperation can sustain or arise in a dynamically evolving network where interactions among agents are based on a SPD but where each agent can decide with whom he will interact. Hanaki et al. (2005) examine the co-evolution and collective behavior using a stochastic learning approach and present an extensive analysis for a wide range of parameter values. Under their co-evolutionary dynamics, they find that high levels of cooperation in large population can be achieved in sparse networks and show that there is an important trade-off between local reinforcement and global expansion. By other side, Eguíluz et al. (2005) enable agents to adapt their initial exogenous local neighborhood according to their satisfaction level and the strategy played. Their dynamics generate a social network that can have the topology of a small world network. Moreover, the network reaches a strong hierarchical structure in which the leaders play an essential role in sustaining a highly cooperative regime. A remarkable point that is a common feature in both co-evolutionary networks and also for the SPD in random networks is the fact that the initial fraction of cooperators in 
the system must be sufficiently high in order to observe an evolutionary network with a higher fraction of cooperators. The argument used in literature is that cooperative behavior will replicate throughout the network, only if the approximate average payoff for agents that cooperate is larger than the one for agents that defect. Thus the interacting dynamics promote higher levels of cooperation in the network only when the initial level of cooperation is high enough.

We are interested in mechanisms that promote higher levels of cooperation for the SPD no matter the initial proportions of cooperators or network setups. For our exploratory analysis, we consider the two dimensional lattice, as an example of high clustered structure, random networks, as the opposite case, and coevolutionary networks, as an important case of hierarchical structure that model real social networks. It can be shown that for the studies reported for the SPD in the lattice the fraction of cooperators falls down from initial high fractions of cooperators. By other hand, from initial fraction of cooperator lower than $1 / 2$ there is a high probability to reach full defecting state in random and adaptive networks. We provide a mechanism based on taxes and subsidies that significantly enhances higher level of cooperations in the lattice for all starting proportions and configurations of cooperators. In the case of random or adaptive networks similar successful results are observed from low initial fraction of cooperators, including the most adverse case of just one cooperative agent in the network. Additionally, our mechanism leads to robust configurations in a finite period of time such that the fraction of cooperators remains unchanged even when the mechanism is switched off. Thus the mechanism can work as an ignition engine to achieve highly cooperative level.

After this brief introduction, we will describe the mechanism in detail. Section 3 and 4 are devoted to study the effects of the mechanism on the lattice, random and adaptive networks. For our analysis we follow the general framework of the SPD presented in Nowak and May (1992), Hanaki et al. (2005) and Eguíluz et al. (2005). For the interaction dynamics in which agents decide with whom they will interact we use the framework in Eguíluz et al. (2005).

\section{A Mechanism of Taxes and Subsidies}

The general framework of the SPD game in a fixed (non adaptive) network is formally described with an action rule performed at each time step that consists in two stages:

Stage 1: Each agent interacts with other partners using the same strategy (C or D) and collecting a total individual payoff according to the PD payoff bi-matrix shown in Table I, where $b>\sigma>\delta>0$ and $b / 2<\sigma$.

Stage 2: Each agent revises his current strategy and updates it by imitating the strategy of his partner with the highest payoff. All strategies are updated at the same time in a synchronous updated process. 
Table I. Prisoners Dilemma payoff bi-matrix.

\begin{tabular}{lll}
\hline & $\mathrm{C}$ & $\mathrm{D}$ \\
$\mathrm{C}$ & $\sigma, \sigma$ & $0, b$ \\
$\mathrm{D}$ & $b, 0$ & $\delta, \delta$ \\
\hline
\end{tabular}

We remark that in stage 1 each agent $i$ simply recollects his total payoff $\Pi_{i}$ which can be defined by

$$
\Pi_{i}=s_{i} \mu_{i} \sigma+\left(1-s_{i}\right)\left(\mu_{i} b+\left(K_{i}-\mu_{i}\right) \delta\right),
$$

where $K_{i}$ is the total number of neighbors of agent $i, \mu_{i}$ is the number of neighbors of agent $i$ that are cooperators, and $s_{i}=0$ or 1 depending on whether agent $i$ is a defector or not.

We want to introduce now a compensation mechanism based on taxes and subsidies in this evolutionary rule by modifying stage 1 . The motivation behind this is to avoid social catastrophes characterized by stationary states with low fractions of cooperators. For expository purposes, let us imagine one individual out of the spatial structure acting as a central planner collecting an involuntary tax to each agent and paying subsidies to reward cooperation. Thus, agent $i$ is taxed with a fixed share $t$ of his total payoff, that is $t \Pi_{i}$, where $0 \leq t<1$. Let $\Pi=\left(\sum_{i} \Pi_{i}\right)$ be the total payoff on the system. The planner rewards $\mathrm{C}$-agents (agents who play cooperation) paying them a subsidy $\beta$ that comes from the recollected taxes. That is, $\beta=t \Pi / C$ where $C$ is the number of cooperator at present time step. Since the parameter $b$ represents the incentive to defect in the PD game, we consider the subsidy $\beta$ as an incentive to cooperate in the network formation. The planner action is performed between stages 1 and 2, where agents are taxed and cooperators receive their compensations. This happens after they play with their partners but before they imitate the strategy of the partner with the highest total payoff. Formally, the total payoff of agent $i$ at present time step in the evolutionary dynamics we are considering is

$$
\Pi_{i}^{\prime}=(1-t)\left(s_{i} \mu_{i} \sigma+\left(1-s_{i}\right)\left(\mu_{i} b+\left(K_{i}-\mu_{i}\right) \delta\right)\right)+s_{i} \beta
$$

It is important to notice that agents are not necessarily awake about the existence of a central planner. In fact, since each agent solely seeks the largest possible benefit from local interactions imitating the strategy of his most successful neighbor what the mechanism does is a straightforward modification of the total payoff recollected by agent $i$ in stage 1 . This total payoff changes from the one defined in equation (1) to the one defined in (2). 


\section{The Mechanism in a Lattice}

We randomly place C-agents and D-agents on a two-dimensional $n \times n(=N)$ square lattice with periodical boundaries. We assume that at least one $\mathrm{C}$-agent must exist in the initial configuration. Let $K$ be the number of immediate neighbors of each agent. The choice of $K=4$ or 8 , depends on what type of neighborhood we are considering, von Newman or Moore respectively. It is easy to see that when the number of cooperators consists on exactly one $\mathrm{C}$-agent the minimal value of the share $t^{*}$ such that the mechanism enhances some level of cooperation is

$$
t>t^{*}=\left(K+1+\frac{(N-(K+1)) \delta K}{b+(K-1) \delta}\right)^{-1}
$$

We observe that $t^{*}$ decreases as $N$ increases. Also, as $\delta \rightarrow 0$ then $t^{*} \rightarrow 1 /(K+1)$ that decreases as $K$ increases.

To make suitable comparisons with previous works, we set $N$ between $10 \times 10$ and $100 \times 100, \delta=0.1$ and $\sigma=1$. The parameter $b$ which controls the incentive to defect varies in the range $1<b<2$. Hence for all values that we are considering, $t^{*}$ varies between 0.0003 and 0.05 . We examine the mechanism setting $t=0.05$, the minimal value of share that enhances some level of cooperation for any $N \geq 100$, $b \in(1,2), K=4$ or 8 and any initial proportion of C-agents.

In a square lattice individuals who always cooperate coexist indefinitely with individuals that always defect showing chaotically spatial patterns that even thought depend on the magnitude of the parameter $b$ it is almost always independent of the initial proportions of $\mathrm{C}$-agents. Moreover, the asymptotic overall fraction of cooperators fluctuates around a relative low value for almost all starting proportions and configurations. For instance, when $N=100 \times 100$ and $K=4$ the asymptotic fraction of cooperators fluctuates around 0.3961 after 100 time steps when we set $b=1.35$ and the initial fraction of $\mathrm{C}$-agents is around or bigger than 0.1. However, for the same initial configurations and parameter values, the mechanism of taxes and subsidies significantly enhances higher proportion on the asymptotic state for all starting proportions and configurations, see Figure 1.

Even though the mechanism always leads to significantly higher proportion of cooperation versus the non-taxed case, we observe how the asymptotic fraction slightly decreases as the initial proportion of cooperators increases. This happens for initial proportions under 0.7. However, on this range the asymptotic fractions are higher than the initial fractions of cooperators. Out of this range, specifically for initial proportions over 0.7 and under 0.9 , the asymptotic fraction slightly fluctuates around 0.7 , after that we note how this behavior changes and the asymptotic fraction increases as the initial proportion of cooperators increases but it does not achieve values higher than the initial fraction. This suggest the existence of thresholds for effective taxation levels that promote higher levels of cooperation than 


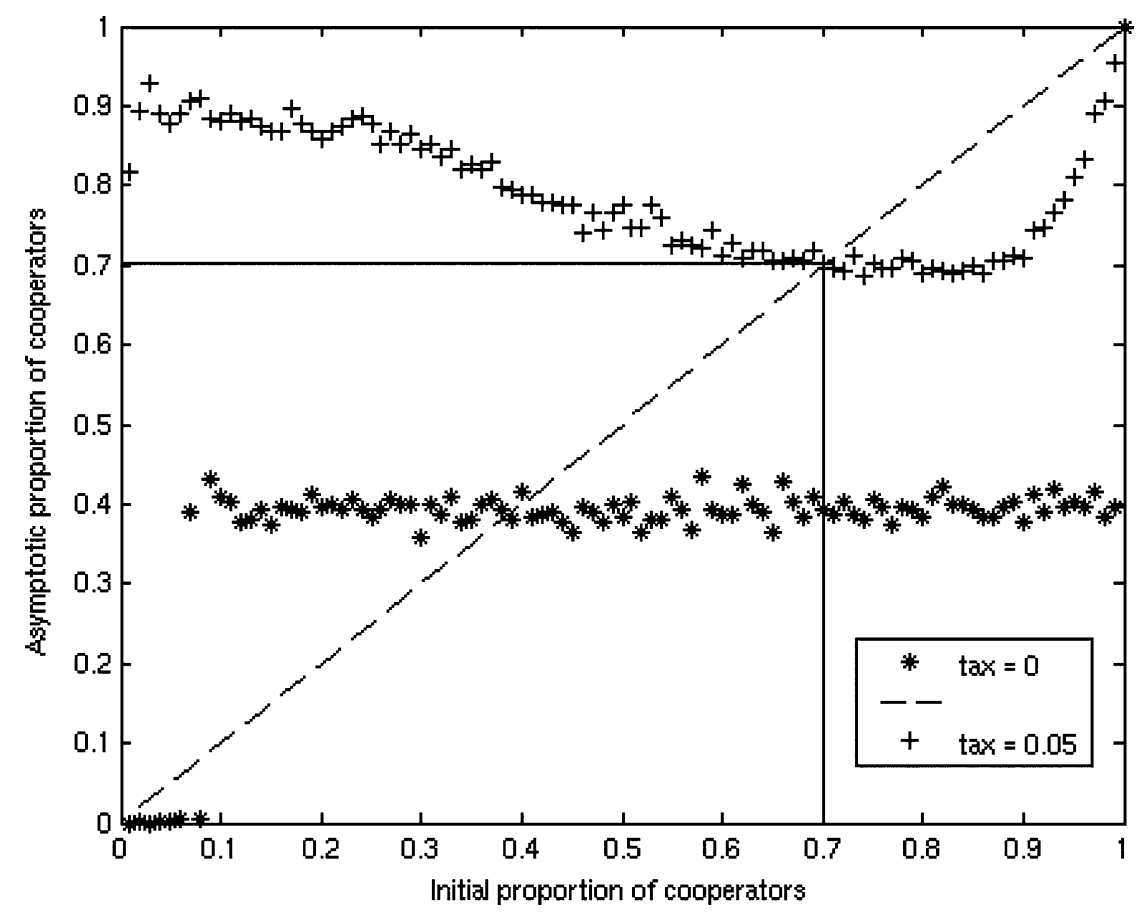

Figure 1. Asymptotic fraction of cooperators as function of initial fraction.

initial ones. In our study case where the share value is 0.05 this threshold value is around 0.7 .

In order to study the robustness of the configuration reached under the mechanism, we switch it off after 200 generations observing that the asymptotic behavior concerning to the fraction of cooperators remains unchanged. Figure 2 shows the stationary spatial patterns after 500 time steps of a non-taxed lattice (left side) and of a taxed lattice (right side) with the same initial proportion of cooperators equal 0.6. The fraction of cooperators chaotically fluctuates around 0.3961 for the non-taxed lattice while it slightly fluctuates around 0.7150 for the taxed lattice.

The stationary regime is reached in both cases at a short period of time. After that, the mechanism can be eliminated without observable changes on the stationary regime. Figure 3 shows the time series of the fraction of cooperators for the nontaxed lattice and taxed lattice during 200 generations.

For low initial numbers cooperators the asymptotical behavior reaches the full defect state in the non-taxed scheme. We show the power of the incentive by examining the asymptotic behavior when we place only 1, 4 and $5 \mathrm{C}$-agents on their respectively initial lattices. In Figure 4 we can observe the spread action of the Cagents for such lattices. In all of them the total reproduction of cooperators reaches around 500 agents. 

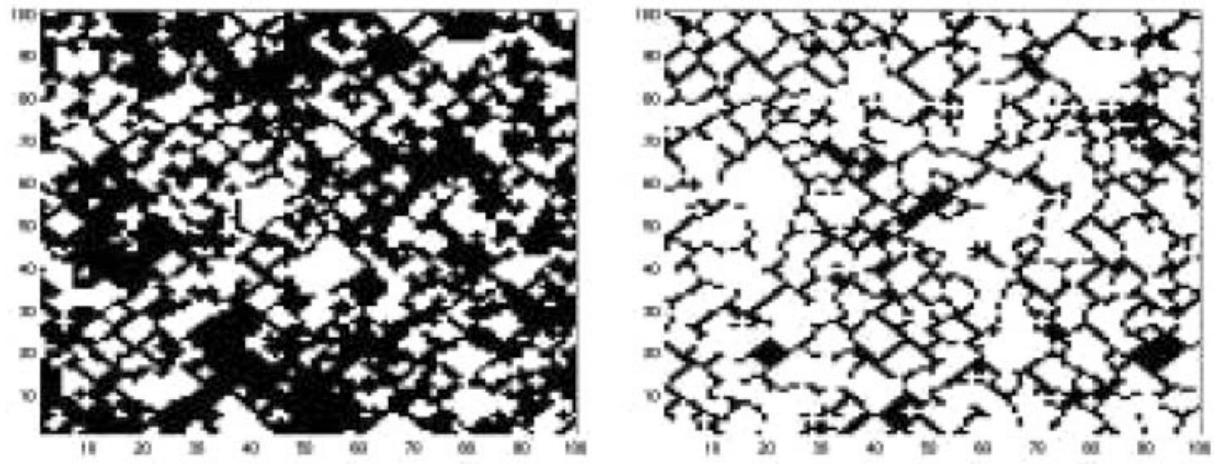

Figure 2. Asymptotic spatial patterns from random initial configuration with $60 \%$ of cooperation for a non-taxed and a taxed lattice.

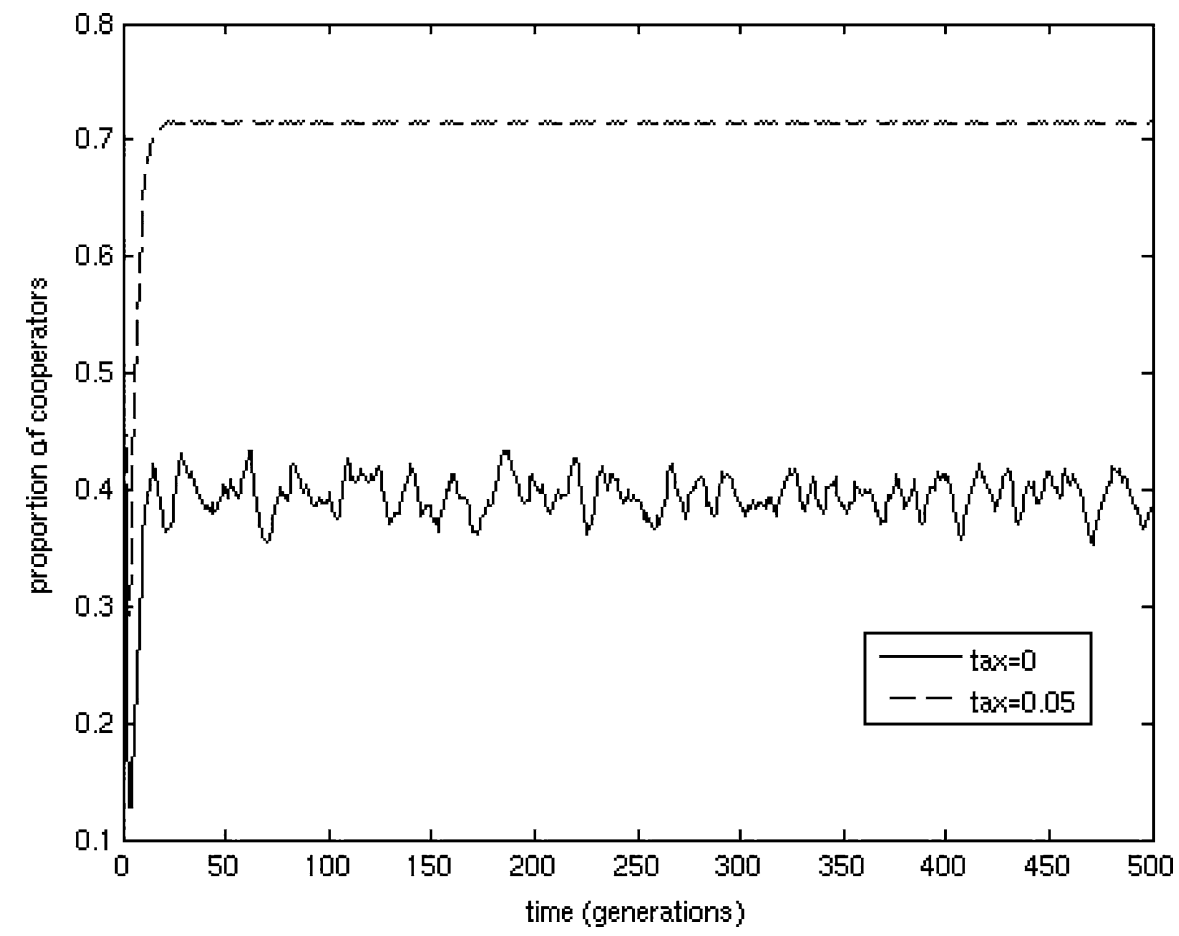

Figure 3. Time series of the fractions of cooperators.

\section{The Mechanism in Random and Adaptive Networks}

In random networks the neighbors for each agent are randomly chosen from the whole network. Therefore $K$ now represents a coordination number defined as the 

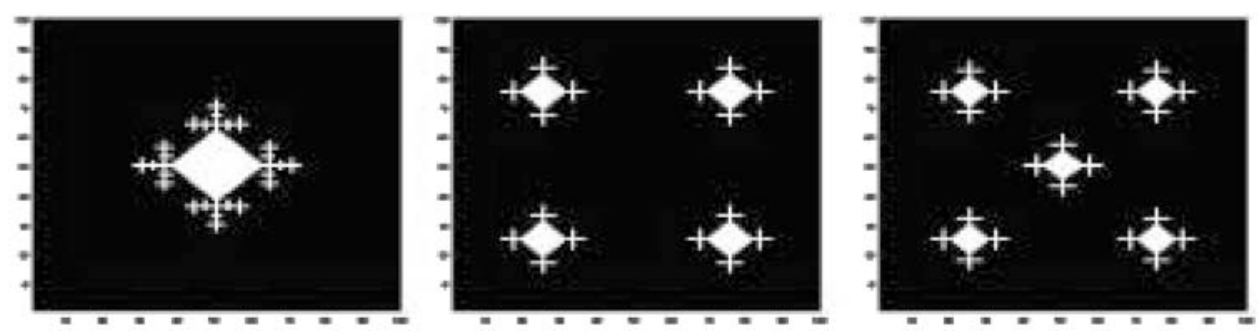

Figure 4. Stationary spatial patterns for initial numbers of 1, 4 and 5 cooperators respectively.

expected average of neighbors per agent,

$$
K=\mathbf{E}\left(\frac{\sum_{i=1}^{N} K_{i}}{N}\right)
$$

where $N$ is the number of agents on the network and $K_{i}$ the number of neighbors of agent $i \in\{1,2, \ldots, N\}$. The number of links in the network, $L=\sum_{i=1}^{N} K_{i} / 2$, is a Binomial random variable with expected value $K N / 2$.

In random networks the fraction of cooperators can either fluctuate slightly or the system can reach a full defect state where all agents play the D strategy. Actually the fraction of cooperators fluctuates slightly when the initial fraction of cooperators in the network is sufficiently high. An initial fraction of 0.6 of C-agents has been used in previous works (e.g Zimmermann et al. (2001) and Zimmermann et al. (2004)) and it is a suitable value for coordination numbers $K=4$ and $K=8$.

For a fixed network size, if the characteristic coordination number $K$ is large enough, there exists a critical value $b^{*}$, which depends on the network structure at initial time, such that for $b>b^{*}$ the system reaches a state of all D-agents and partial cooperation is supported for $b<b^{*}$. In Table II, we consider $N=100$, and the averages over 10 random initial networks. We observe that for the 10 studied cases, $K=4$ was not large enough to observe $b^{*}>1$. However, for $K=8$, we observed the existence of critical values. Table II showed that the average of theses critical values lied in $(1.55,1.75)$.

In the tax scheme, we did not observe a critical $b^{*}$ for any $K=4$ or $K=8$. As in previous section we set $t=0.05$. The network with exactly one $\mathrm{C}$-agent at initial time never reaches a full defect state. The fraction of cooperators is quite robust from increasing value of the incentive to defect $b$ and from increasing value of the average number of links per agent $K$. It is important to remark that by (3) $t^{*}$ decreases as $N$ increases then for greater sizes of the network we can find lower values of $t$ such that the mechanism is still working.

We illustrate in Figure 5(a) the asymptotic dynamics of the fraction of cooperators with a time series of $f_{C}$ evolving for a non-taxed network $(t=0)$, and for a taxed network $(t=0.05)$. We observe that the fraction of cooperators fluctuates 
INCENTIVES TO COOPERATE IN NETWORK FORMATION

Table II. Averages of the fractions of C-agents in random networks.

\begin{tabular}{|c|c|c|c|c|c|c|c|c|}
\hline \multirow[b]{3}{*}{$b$} & \multicolumn{4}{|c|}{$0.6 \mathrm{~N} \mathrm{C}$-agents at initial time } & \multicolumn{4}{|c|}{ One $\mathrm{C}$-agent at initial time } \\
\hline & \multicolumn{2}{|c|}{$K=4$} & \multicolumn{2}{|c|}{$K=8$} & \multicolumn{2}{|c|}{$K=4$} & \multicolumn{2}{|c|}{$K=8$} \\
\hline & $t=0$ & $t=.05$ & $t=0$ & $t=.05$ & $t=0$ & $t=.05$ & $t=0$ & $t=.05$ \\
\hline 1.05 & 0.920 & 0.944 & 0.972 & 0.992 & 0 & 0.937 & 0 & 0.968 \\
\hline 1.15 & 0.887 & 0.910 & 0.710 & 0.929 & 0 & 0.948 & 0 & 0.923 \\
\hline 1.35 & 0.626 & 0.789 & 0.325 & 0.754 & 0 & 0.753 & 0 & 0.652 \\
\hline 1.55 & 0.322 & 0.457 & 0.057 & 0.383 & 0 & 0.596 & 0 & 0.489 \\
\hline 1.75 & 0.177 & 0.352 & 0.000 & 0.238 & 0 & 0.375 & 0 & 0.219 \\
\hline 1.95 & 0.046 & 0.264 & 0.000 & 0.215 & 0 & 0.198 & 0 & 0.155 \\
\hline
\end{tabular}

(a)

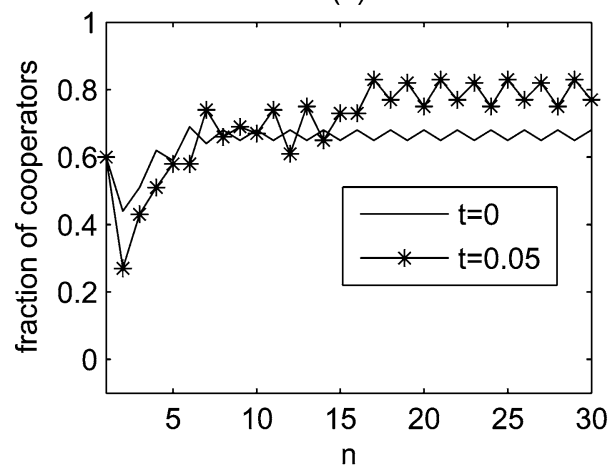

(c)

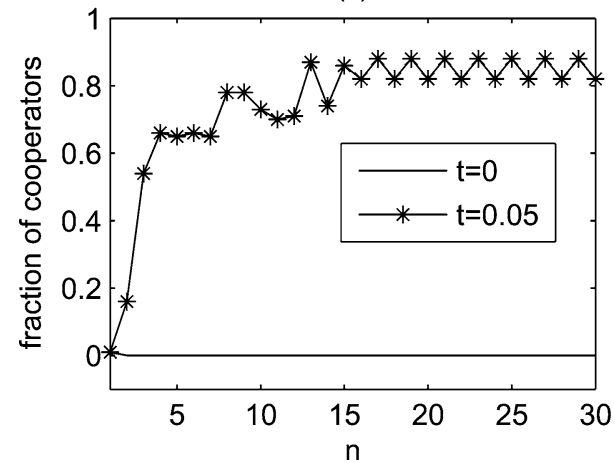

(b)

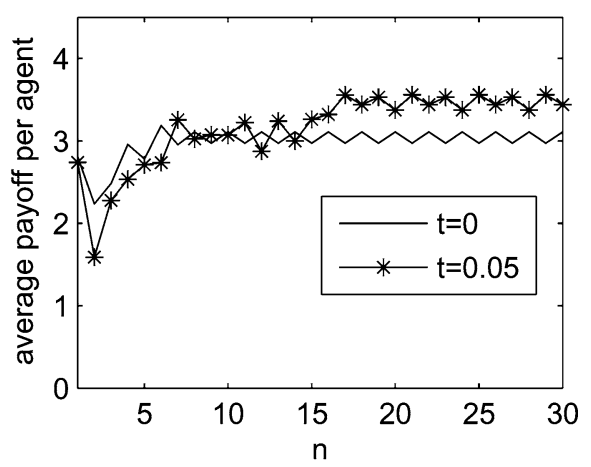

(d)

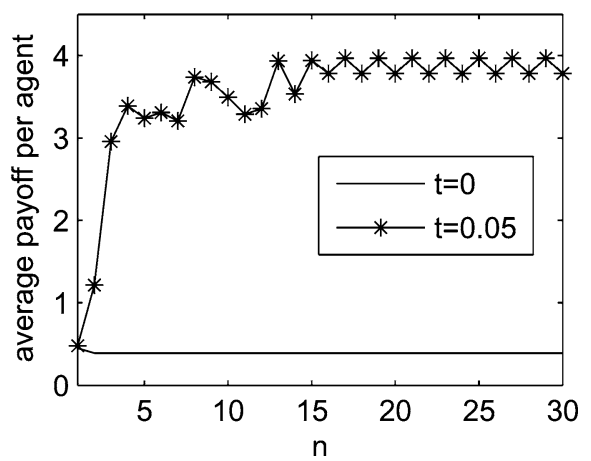

Figure 5. Time series of $f_{C}$ and $\Pi / N$ in full non-adaptive networks.

slightly after some transient time for both cases (tax and non-tax). Here, the initial fraction of C-agents is 0.6 and $b=1.35$. Table II illustrates this behavior for other values of $b$. In the case of an initial network of solely one C-agent, Figure 5(c) shows an extremely different result between taxes and non-taxes case. In the 
(a)

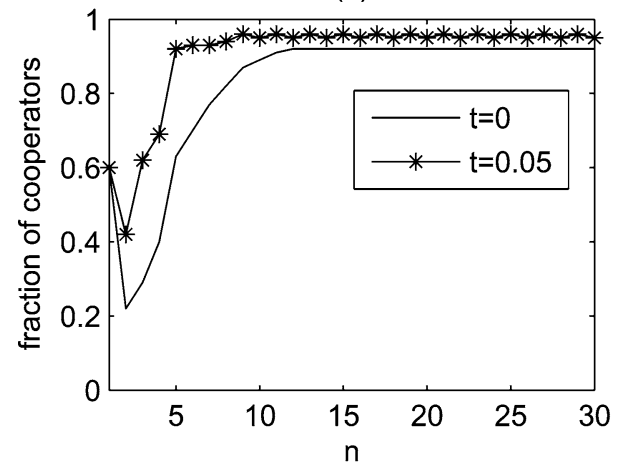

(c)

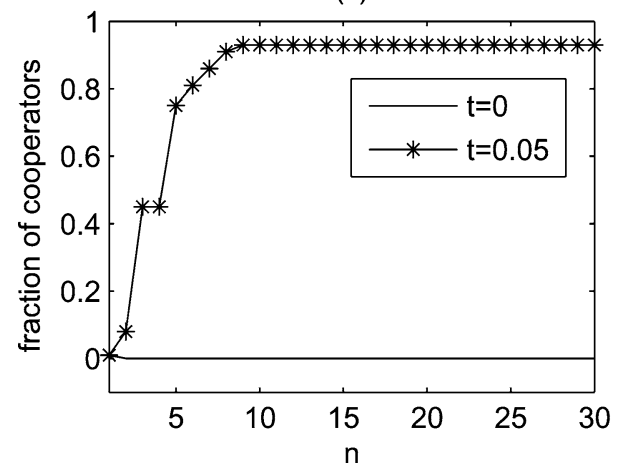

(b)

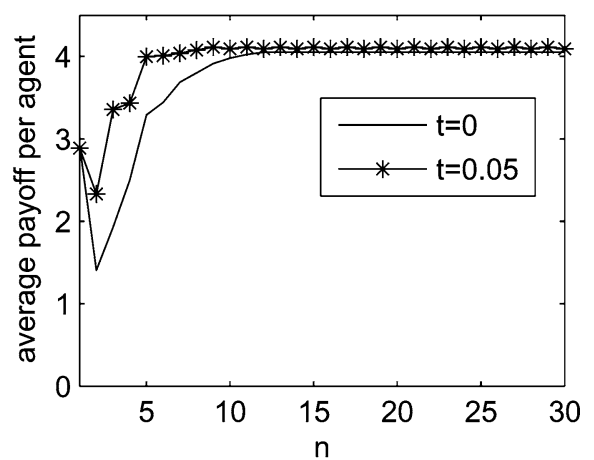

(d)

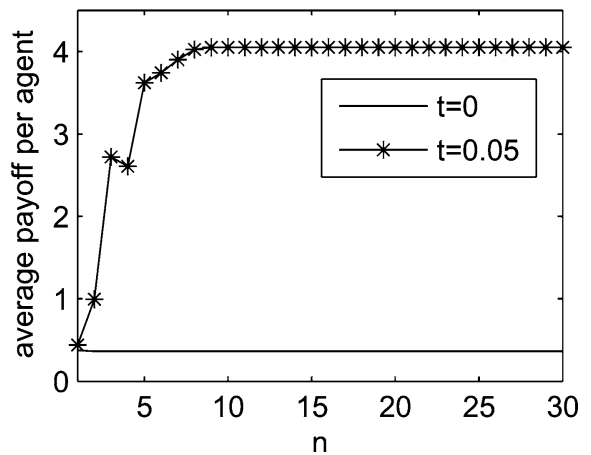

Figure 6. Time series of $f_{C}$ and $\Pi / N$ in full adaptive networks.

non-taxes case, the only cooperator switches to defect and a full defective network is reached, in contrast in the taxes case, the mechanism enhances a highly cooperative network. The mechanism of taxation preserves the dynamics that settles onto a fluctuated fraction of cooperators after some transient time starting when the fraction of cooperators reaches a value greater than 0.6.

Figure 5 (b) and (d), show the effect that the mechanism of taxation has over the average payoff of the whole network. When the initial fraction of $\mathrm{C}$-agents is 0.6 , the mechanism produces a slight effect over the average of payoff per agent. However, this mechanism has a strong influence in the social macrostructure of network with a small initial fraction of cooperators because not only cooperators want to keep cooperating but also defectors switch to imitate successful cooperators producing an increasing average payoff per agent.

Now we consider the co-evolutionary dynamics discussed in the sequel (Eguíluz, et al. (2005), Zimmermann et al. (2001) and Zimmermann et al. (2004)). The stage evolution of this adaptive network is as follows:

Stage 3: Each D-agent may adapt its local neighborhood. This is performed if a D-agent is an unsatisfied agent, that means he does not have the highest payoff. 
INCENTIVES TO COOPERATE IN NETWORK FORMATION

Table III. Averages of the fractions of C-agents of full adaptive networks.

\begin{tabular}{|c|c|c|c|c|c|c|c|c|}
\hline \multirow[b]{3}{*}{$b$} & \multicolumn{4}{|c|}{$0.6 \mathrm{~N} \mathrm{C}$-agents at initial time } & \multicolumn{4}{|c|}{ One $\mathrm{C}$-agent at initial time } \\
\hline & \multicolumn{2}{|c|}{$K=4$} & \multicolumn{2}{|c|}{$K=8$} & \multicolumn{2}{|c|}{$K=4$} & \multicolumn{2}{|c|}{$K=8$} \\
\hline & $t=0$ & $t=.05$ & $t=0$ & $t=.05$ & $t=0$ & $t=.05$ & $t=0$ & $t=.05$ \\
\hline 1.05 & 0.951 & 0.966 & 0.898 & 0.995 & 0 & 0.968 & 0 & 0.996 \\
\hline 1.15 & 0.943 & 0.943 & 0.697 & 0.992 & 0 & 0.942 & 0 & 0.996 \\
\hline 1.35 & 0.819 & 0.948 & 0.395 & 0.884 & 0 & 0.920 & 0 & 0.990 \\
\hline 1.55 & 0.526 & 0.777 & 0.196 & 0.785 & 0 & 0.812 & 0 & 0.685 \\
\hline 1.75 & 0.443 & 0.639 & 0.100 & 0.578 & 0 & 0.630 & 0 & 0.598 \\
\hline 1.95 & 0.346 & 0.514 & 0.000 & 0.371 & 0 & 0.335 & 0 & 0.396 \\
\hline
\end{tabular}

Then with probability $p$, he breaks each link with each of his D-neighbor, and will replace it with a new agent randomly chosen from network.

With this adaptive rule the coordination number $K$ remains constant: for each unsatisfied D-agent, it will replace on average D-neighbors by new neighbors randomly chosen from the whole network, and thus its number of neighbors $K_{i}$ will not change. The total number of links in the network is conserved since the replaced D-agents will lose one link and the new selected ones will gain one link. Therefore, a $\mathrm{C}$-agent may increase its number of neighbors by receiving new links from searching D-agents. Destruction or spontaneous creation of links are not taking into account in this model. In order to make suitable comparisons with results presented in Eguíluz et al. (2005); Zimmermann et al. (2001) and Zimmermann et al. (2004), we consider as well random initial network with coordination number $K=4$ and $K=8$ and full adaptive network $p=1$.

Table III shows the averages of the fractions of C-agents, at the steady state, of full adaptive networks $(p=1)$ with and without taxation. The averages are taking over 10 different initial conditions after 30 time steps of evolution.

First, let us examine the case of initial $0.6 \mathrm{~N} \mathrm{C}$-agents without taxation $(t=0)$. We can observe how the average of the fractions of $\mathrm{C}$-agents, at the steady state, decreases with an increasing value of the incentive to defect. The numerical results also show that increasing the coordination number, $K$, the average fraction of $\mathrm{C}$-agents decreases faster with $b$. Now, if we apply the mechanism of taxes and subsidies described above with $t=0.05$, we observe that an increasing value of the average number of links per agent $K$, does not significantly decrease the average fraction of $\mathrm{C}$-agents. That means, the mechanism is quite robust from changes of $K$.

As we can see, the most remarkable result occurs in networks with only one $\mathrm{C}$-agent in the initial time step. We observe how the mechanism of taxes and 
subsidies has a successful effect in such networks, the initial cooperator positively responds to the incentive to keep cooperating and produces the effect for defectors to change their strategies. The mechanism enhances highly cooperative networks for both initial fractions of C-agents.

Figure 6 illustrates the asymptotic behaviors of taxed and non-taxed networks for $b=1.35$ and $K=4$. We show in Figure 6 (a) the time series of $f_{C}$ with initial fraction of $0.6 \mathrm{~N} \mathrm{C}$-agents. We observe that the fraction of cooperators increases in both cases (taxed and non-taxed). Similar results were observed for the different values of $b$ and $K$ considered in Table III. We conclude that the taxation enhances a highly cooperative network comparable with the non-taxed scheme. In the case of initial one $\mathrm{C}$-agent, the results are extremely different between taxes and non-taxes case. Figure 6 (c) shows how the only cooperator switches to defect and a full defective network is reached in the non-taxes case, in contrast with the taxes case, where the mechanism enhances a highly cooperative network. The mechanism of taxation not only preserves the dynamics that settles onto a steady state after some transient time, also preserves the properties described in Eguíluz et al. (2005) such as the emergence of a leader and a cooperator with maximum number of connections.

The graphics (b) and (d), in Figure 6, show the effect that the mechanism of taxation has over the average payoff of the whole network.

Although the mechanism could be seem to be socially unfair, because all agents are taxed but only $\mathrm{C}$-agents receive the incentive, the increasing number of cooperators makes the incentive negligible and dispensable at short time. The taxation may be used as an ignition engine in networks with low initial fraction of $\mathrm{C}$-agents. This mechanism of redistribution of wealth has a strong influence in the social macrostructure of the network because not only cooperators want to keep cooperating but also defectors want to change their strategies to cooperate.

\section{Concluding Remarks}

In this paper, we have proposed a mechanism of taxes and subsidies to arise high cooperation in the SPD game. We examined the mechanism in different wellstudied structures as lattice, random and adaptive networks. Our results show that the mechanism works as an ignition engine to achieve highly robust cooperative level for any value of the incentive to defect. When the initial structure consists of just one cooperative agent, the mechanism not only allows the coexistence of cooperators and defectors but also can enhance a highly cooperative state for a suitable taxation level contrasting with the results in the non-taxes scheme. Under the mechanism of taxation the studied systems reach stationary states at short period of time that remain invariants even when the mechanism is turned off.

We finally remark that the mechanism described can be easily explained in an economic or social context where we can assume the existence of a benevolent central planner who redistributes wealth among agents searching for improving 
collective outcomes in the long-run time of this network formation. The idea here is not to discuss over the existence or not of such planner, we just explore a solution to avoid inefficient macrostructure in evolutionary networks.

\section{Acknowledgment}

The authors are grateful to the anonymous referee for his useful comments.

\section{References}

Abramson, G. and Kuperman, M. (2001). Social games in a social network. Phys. Rev. E, 63, 030901.

Doebeli, M. and Hauert, Ch. (2005). Models of cooperation based on the Prisoner's Dilemma and the Snowdrift game. Ecology Letters, 8, 748-766.

Eguíluz, V.M., Zimmermann, M.G., Cela-Conde, C.J. and San Miguel, M. (2005). Cooperation and the emergence of role differentiation in the dynamical of social networks. Amer. J. Soc. 110, 977-1008.

Hanaki, N., Peterhansl, A., Dodds, P.S. and Watts, D.J. (2005). Cooperation in evolving social networks. Working Paper.

Lindgren, K. and Nordahl, M.G. (1994). Evolutionary dynamics of spatial games. Physica D, 75, 292-309.

Nowak, M. and May, R. (1992). Evolutionary games and spatial chaos. Nature, 359, 826-8829.

Nowak, M. and May, R. (1993). Spatial dilemmas of evolution. Int. Adv. Complex Syst. 5, 35-78.

Vriend, N. (2005). ACE Models of Endogenous Interactions. To appear in K.L. Judd and L. Tesfatsion (Eds.), Handbook of Computational Economics. Volume 2: Agent-Based Computational Economics., North-Holland .

Zimmermann, M., Eguíluz, V. and San Miguel, M. (2001). Cooperation, adaptation and the emergence of leadership. In A. Kirman and J-B Zimmermann (Eds.), Economics with Heterogeneous Interacting Agents. Lecture Notes in Economics and Mathematical Systems, N503, 73-86. Springer.

Zimmermann, M.G., Eguíluz, V.M. and San Miguel, M. (2004), Coevolution of dynamical states and interactions in dynamic networks. Phys. Rev. E, 69, 065102. 